\title{
Acceso temático a los contenidos de las colecciones de bibliotecas de la UNAM: historia y perspectivas
}

\author{
Catalina Naumis Peña *
}

Artículo recibido:

16 de noviembre de 2011.

Artículo aceptado:

11 de junio de 2012.

\section{RESUMEN}

En general el análisis de las decisiones científicas del pasado permite obtener una mirada más objetiva frente a las consecuencias y ayuda a visualizar mejores soluciones en el futuro. En el caso del acceso temático a las colecciones bibliográficas de la UNAM desde su creación se observan influencias culturales en los instrumentos lingüísticos para indizar y recuperar, que se mantienen hasta el día de hoy. Se resalta la conveniencia de analizar las decisiones instrumentadas a la luz de las propuestas de acceso temático en el presente. Por lo tanto, en el trabajo se revisan principios sustentados desde una concepción socio-cognitiva del tratamiento terminológico que indican la conveniencia de

* Instituto de Investigaciones Bibliotecológicas y de la Información de la UNAM, México.naumis@unam.mx

INVESTIGACIÓN BiBLIOTECOLÓGICA, Vol. 26, Núm. 57, mayo/agosto, 2012, México, ISSN: 0187-358X. pp. 177-198 
adoptar metodologías de trabajo, en el acceso temático a la información, con un pensamiento de respeto hacia la cultura transmitida a través de las lenguas, a pesar del costo elevado que ello supone.

Palabras clave: Índices temáticos; Clasificación bibliográfica; Historia del acceso temático; Bibliotecología y Terminología; Bibliotecología y Traducción; Perspectiva socio-cognitiva del acceso temático.

\section{ABSTRACT}

Subject access to the contents of UNAM library collections: history and perspectives Catalina Naumis Peña

Overall analysis of the scientific decisions of the past allows a more objective look in regard with with the consequences and helps as to visualize better solutions in the future. In the case of subject access to library collections at UNAM since its settlement, cultural influences are seen in the linguistic tools to index and retrieve it, which remain until today. This paper highlights the convenience of analyzing the decisions considering present subject access proposals. Therefore we review the principles based on a conception of social-cognitive processing terminology indicating the convenience of working methods in the subject access to information, and with respect for the culture transmitted through the languages, despite the high cost that entails.

Keywords: Subject indexes; Bibliographic classification; History of Mexican libraries; Library Sciences and Terminology; Library Sciences and Translation; Socio-cognitive perspective in the subject access. 
"Imitar si no se puede hacer otra cosa; pero
aun al imitar, inventar un tanto, adaptar"...
El verdadero redentor no es el iluso
que desconoce el suelo donde pisa,
sino el sabio que combina lo real y lo ideal
en proporciones armoniosas

Antonio Caso, 1943

— l objetivo de este trabajo es destacar el proceso que llevó a definir las po- líticas de acceso temático en las bibliotecas de la Universidad Nacional Autónoma de México (UNAM) haciendo hincapié en las primeras décadas del siglo XX, abarcando desde su creación hasta la época actual para analizar las influencias culturales que se observaron en ellas y las consecuencias al día de hoy, tomando en cuenta que las soluciones bibliotecológicas no pueden desligarse del desarrollo político, social y económico de un país. En la actualidad el Sistema Bibliotecario de la UNAM reporta 134 unidades de información, cuyas colecciones están ordenadas por un sistema de clasificación y además ofrecen un acceso temático calificado, con base en instrumentos lingüísticos recomendados por los organismos internacionales de normalización bibliotecaria.

\section{Planteamiento}

A los efectos de establecer un marco para entender el acceso temático a las colecciones, es decir, el modo como el usuario puede localizar los documentos que responden a una necesidad de información en una temática específica del conocimiento, se explica el resultado del proceso de indización (catalogación y asignación de una clasificación bibliográfica) de los recursos documentales en los acervos.

La indización se realiza a través de un conjunto de procedimientos definidos de antemano para crear puntos de acceso a los contenidos de los registros del conocimiento, con el propósito de recuperación por los usuarios de información. Se obtienen índices por cada punto de acceso como puede ser el autor de una obra, un título u otras expresiones, que llevan al usuario hacia el documento que trata de localizar.

En este trabajo se estudia el punto de acceso temático, ya sea a través de la catalogación por materias o la clasificación. El común denominador de la representación temática es la utilización de: palabras significativas simples o compuestas, términos científicos o tecnológicos, símbolos numéricos o alfanuméricos, validados en un proceso de normalización, como en el caso de los descriptores, encabezamientos de materia o palabras clave. Los símbolos numéricos 
o alfanuméricos son extraídos de esquemas de clasificación donde se encuentra la equivalencia con la designación lingüística en un orden sistemático por campos del conocimiento. La utilización de signos lingüísticos para calificar la información está basada en la función comunicativa de la lengua (Cabré, 1999, 71).

La investigación en catálogos, búsqueda bibliográfica y archivística sobre la que se basa el presente trabajo estuvo enfocada a documentar cuáles fueron y son los puntos de acceso utilizados, para conocer los contenidos temáticos de los libros (catalogación por materias) y el orden por campos del conocimiento (clasificación bibliográfica) utilizados en las diferentes colecciones de libros que poseen las bibliotecas de la UNAM.

El énfasis en la catalogación por materias y en la clasificación (los dos aspectos de contenido comprendidos en la indización) son los categorizadores de información, en donde existe una buena dosis de subjetividad y, por lo tanto, donde se reflejan las influencias culturales. Decía Escamilla: "La asignación de un número de clasificación apropiado y la de los encabezamientos de materia pertinentes requiere esencialmente de la misma actividad intelectual por parte del catalogador" (Escamilla, 1970, p. 181).

Es difícil separar en una historia del acceso temático los aspectos generales de la organización documental. Tampoco es posible separar la historia de la organización documental entre bibliotecas públicas y de la UNAM en los primeros años del siglo XX, porque se trabaja con sistemas imbricados entre las mismas.

Los antecedentes cercanos a la creación de la Universidad Nacional y el desarrollo de sus bibliotecas muestran la relación de los intelectuales mexicanos que intervinieron en los proyectos bibliográficos internacionales surgidos en Europa y se parte del supuesto en este trabajo que las influencias culturales externas penetraron con tal intensidad en el acceso temático a las colecciones, a través de la indización y la clasificación adoptada en las bibliotecas, que impidieron un desarrollo propio, observado aún en la actualidad.

En 1899 se crea en México el Instituto Bibliográfico Mexicano ligado al ambicioso proyecto de la Royal Society of London, su International Catalogue of Scientific Literature y además asiste Francisco del Paso y Troncoso a los dos congresos internacionales celebrados por la sociedad mencionada. Estas colaboraciones y participaciones colocan a México en una posición privilegiada frente al resto de las naciones de habla hispana (Astrain, Olagûe y Menéndez, 2001, p. 302) en relación a la organización de sus fondos bibliográficos.

Otra relación importante, a nivel de bibliotecas y archivos, es la establecida con el Instituto Internacional de Bibliografía de Bruselas que se concreta en la creación de la oficina de Bibliografía Mexicana. La Biblioteca Nacional 
dirigida por José María Vigil adopta la clasificación de Paul Namur, bibliotecario belga.

Durante el gobierno porfirista (1876-1911), el positivismo era la corriente filosófica dominante en la ciencia nacional, como elemento vertebrador de la sociedad mexicana explicando así los esfuerzos relacionados con la internacionalización de la actividad bibliográfica. El contraste con el censo de población de 1900 era evidente, más del $87 \%$ de la población vivía en zonas rurales, sin el mínimo acceso a la instrucción más rudimentaria, todos los esfuerzos educativos y culturales porfiristas estaban dedicados a la Ciudad de México. En el cambio de siglo, el 80\% de los habitantes mayores de 10 años no sabían siquiera leer ni escribir (Quintana Pali, 1988, p. 17). Sin duda la actitud ante la enseñanza y la cultura es reflejo de los modelos imperantes en la época, matizados por los intereses políticos y personales de quienes asumían las responsabilidades públicas.

\section{Antecedentes históricos}

El 22 de septiembre de 1910 comienza sus actividades la Universidad Nacional. Fue inaugurada en el marco de los festejos del Centenario de la Independencia por el presidente de México, el Gral. Porfirio Díaz. La creación de la universidad fue alentada y promovida por Justo Sierra Méndez, profesor de la Escuela Nacional Preparatoria y Secretario del Ministerio de Instrucción Pública y Bellas Artes, con la idea de contar con una institución mexicana que estudiara y enseñara los valores culturales propios de la sociedad que se consolidaba.

Justo Sierra era un hombre que por su pensamiento no comulgaba con los principios de la élite política del gobierno en el que colaboraba, pero su interés por conseguir el acceso poblacional a la educación era tal, que permaneció en el gobierno hasta que consiguió instalar la universidad. Es famoso su discurso de rompimiento con el pensamiento positivista expresado la noche del veinte de junio de 1908, porque marca el inicio de un movimiento cultural, en el que varios intelectuales coinciden sobre la necesidad del predominio de la vida espiritual sobre la vida material, para conformar el grupo del Ateneo de la Juventud, cuyo liderazgo es asumido por Antonio Caso. Es importante destacar que, impulsado quizás por el afán de adaptar las ideas a la realidad mexicana, hace a un lado la colaboración con la Royal Society of London y los congresos que organiza ésta, a pesar de contar con presupuesto especial para ello. Es decir, su preocupación era resolver el problema cultural a nivel país. 
Las primeras décadas del siglo fueron años de muchos desafíos, entre ellos la consecución de la autonomía universitaria y la conformación de la institución nacional de mayor nivel educativo, una amplia gama de intereses en conflicto que no se pueden enfrentar aislados, sino como un conjunto de problemas relacionados entre sí cuyas soluciones provienen de un plano congruente de planteamientos globales. Es una época de grandes sobresaltos y giros políticos que seguramente repercutieron en las prioridades de la nueva institución que fundó sus expectativas en la acumulación de acervos, para lograr la mejor y más amplia difusión de la cultura a través de los impresos.

En relación a ese periodo del siglo veinte, Morales expresa la situación con estas palabras: "Las escuelas, las bibliotecas, la lectura y las bellas artes fueron concebidas como elementos que fortalecían las relaciones y satisfacen necesidades fundamentales del ser humano; por lo tanto, en ese período, se buscó una fuerte relación entre el hombre, el libro y la lectura, cuyo eje estaba constituido por la educación y dos apoyos fundamentales: la escuela y la biblioteca; la educación era presente y futuro y, a través de la lectura, se podía aspirar a modelos superiores" (Morales, 2010, p. 3).

El apoyo a la educación y la lectura ocurrido en los primeros años del siglo XX es herencia de tradiciones que vienen desde los tiempos posteriores a la conquista. Se cree que México fue el primer país en América Latina que creó oficialmente una biblioteca, en la Catedral de México en 1534, y también el primero en contar con una imprenta, la de Juan Pablos, en 1539. En 1551 se creó la Real y Pontificia Universidad de México y posteriormente se publicó la bibliografía nacional (Bibliotheca Mexicana, Eguiara y Eguren, 1755). Sin duda, los siglos anteriores a la creación de la Universidad Nacional están influidos por la cultura peninsular y la costumbre de acopiar literatura para adquirir conocimiento. La literatura no era de uso generalizado por la población, hasta en las primeras décadas del siglo XX.

\section{CLASIFICACIÓN Y CATALOGACIÓN POR MATERIAS EN MÉXICO}

El interés por los libros se manifestaba en la acumulación de los mismos, pero no se acompañaba de una preocupación por la organización bibliográfica o por lo menos existe muy poco escrito al respecto. No existen datos de una catalogación por materias a principios del siglo XX que representen los contenidos de los libros, si bien existen documentos que permiten afirmar que se había generalizado el Sistema de Clasificación Decimal de Melvil Dewey introducido al país a fines del siglo anterior, en las principales bibliotecas capitalinas. Este sistema condensaba el conocimiento humano en una estructura 
taxonómica. De los sistemas de clasificación anteriores se tiene noticia acerca del catálogo sistemático de la Biblioteca Nacional publicado por José María Vigil que incluía la clasificación con el sistema de Paul Namur modificado en parte y estaba agrupado en diez divisiones temáticas (Castañón, 1974, p. 28). También es conocida la tarea que acometió Juan B. Iguíniz, a la muerte de Vigil, de sustituir paulatinamente los catálogos de libros por tarjetas bibliográficas y de elaborar el catálogo onomástico y el catálogo diccionario (Astrain, Olagûe y Menéndez, 2001, p. 312).

En 1900 la Secretaría de Fomento había publicado un folleto explicativo del Sistema de Clasificación de Melvil Dewey. La biblioteca de la Escuela Nacional Preparatoria había sido fundada en 1879 con algunos de los libros que pertenecieron al convento de San Ildefonso, más el agregado posterior de donaciones; los primeros datos que existen sobre su organización bibliográfica los expone Morales explicando que en 1907 se había adoptado el Sistema Decimal de Dewey para clasificar la colección (Citado de Díaz y de Ovando y García Barragán por Morales, 2010, p. 6).

También en 1907 se publica en España "El catálogo de conceptos en las bibliotecas públicas", como consta en la bibliografía elaborada por Mantecón acerca de reglas y listas de encabezamientos (Mantecón, 1975, p. 244), pero no se tiene noticia de que haya sido usado en las bibliotecas mexicanas para calificar los contenidos de las colecciones.

$\mathrm{El}$ antecedente de esta lista de conceptos se remite a las 91 Reglas de Catalogación de Panizzi elaboradas para la Biblioteca del Museo Británico en 1841, concebidas para recuperar por autor personal, o por entidad y título tanto en libros impresos, como mapas y música. Pocos años después de la aparición de estas reglas, en el año 1848 el bibliotecario William Frederick Poole en Estados Unidos comienza a trabajar en la realización de índices y aconseja que en los catálogos de las bibliotecas, cuando "la materia de un libro no sea expuesta por el autor o a través del título o del texto en una forma clara o usual, el bibliotecario debe señalar la forma equivalente que sí sea clara o usual" (Castañon, 1974, p. 43). La política del uso de términos para representar los temas de los fondos bibliográficos de las bibliotecas públicas mexicanas a principios del siglo XX estaba destinada a apoyar la creación de los catálogos diccionarios, propuestos por el norteamericano Charles A. Cutter en 1876 y que incluían la indización por autor, título y materia.

La siguiente noticia sobre una indización de colecciones también se refiere a la implantación de la Clasificación Decimal de Dewey y esta vez en las Secretarías de Fomento y de Comunicaciones y Obras Públicas en el año 1911. Para ello se contó con una comisión de especialistas de los Estados Unidos de Norteamérica que vino a México y que trabajó en la formación de un 
catálogo alfabético de autores y otro de obras clasificadas en 67 materias, con la indicación del número del estante y del anaquel o entrepaño o sección en que fueron colocados los volúmenes de que se componen dichas obras (Manuel Francisco Álvarez citado por Quintana Pali, 1988, p. 35). Ezequiel A. Chávez fue líder también en esta empresa de organización archivística, sobre todo con las modificaciones propuestas por el Instituto Bibliográfico de Bruselas a la Clasificación Decimal de Dewey. ${ }^{1}$

Como se ha venido anotando en este trabajo, en los primeros años de la creación de la universidad existían

varias personas conocedoras y amantes de las bibliotecas, que impulsaron el desarrollo técnico de éstas y promovieron la idea de que era necesario elaborar criterios uniformes y únicos para la organización de todas las bibliotecas del país. Destacan entre ellos Ezequiel A. Chávez, Luis G. Urbina, Agustín Loera y Chávez y Juan B. Iguíniz (Quintana Pali, 1988, p. 35).

Sin duda Ezequiel A. Chávez ostentó a lo largo de su vida altas responsabilidades y en todas ellas apoyó las bibliotecas. Cuando trabajó de 1903 a 1911 en la entonces Secretaría de Estado y el Despacho de Instrucción Pública y Bellas Artes con Justo Sierra estudia la organización de las universidades de los Estados Unidos. Esta experiencia será fundamental en la elaboración de la ley constitutiva de la Universidad Nacional en 1910.

Ezequiel A. Chávez se convirtió en un propulsor del sistema Dewey. Propició también su utilización para organizar archivos, con las reformas propuestas a Melvil Dewey por Paul Otlet y Henri La Fontaine en el esquema adaptado del Manual del Repertorio Bibliográfico Universal en 1905, que posteriormente se convirtió en la Clasificación Decimal Universal.

Como director de la Escuela Nacional de Altos Estudios en 1913 promueve el desarrollo bibliotecario en la misma, que ya utiliza el Sistema de Clasificación de Melvil Dewey y se sabe que los encargados de algunas de las bibliotecas públicas dependientes de la Secretaría de Instrucción Pública recurrieron al bibliotecario de la misma para solicitar instrucción con la finalidad de implantar el sistema decimal y los procedimientos modernos de

1 Carta del Secretario General de la Unión de Asociaciones Internacionales en Bruselas a Ezequiel A. Chávez en la que se manifiesta estar interesado en las conferencias que ha dictado sobre clasificación decimal, en las publicaciones relativas a la conexión existente entre el Instituto Internacional de Bibliografía y la Unión expresada, y sobre los trabajos presentados en Europa, a iniciativa de ese instituto, para la aplicación de la clasificación en trabajos administrativos. Fondo Ezequiel A. Chávez, Sec.: Secretaría de Comunicaciones y Obras Públicas (Asesoría), Subsec.: Administración y Proyectos, Ser.: Correspondencia. Fecha: 16 de febrero de 1914. 
catalogación y registro (Boletín de Instrucción Pública, t. 21, no. 3 y 4, marabril 1913, p. 323, citado por Quintana Pali, p. 38). Chávez tuvo otras responsabilidades muy altas en la enseñanza y desde los puestos que ocupaba continuó apoyando las bibliotecas universitarias.

Entre otros, de los pocos y primeros antecedentes que se obtienen sobre organización bibliotecaria a principios del siglo XX, se cuenta con la solicitud en 1913 para crear un Departamento de Clasificación y Catalogación en la Biblioteca Nacional, siendo su director Luis G. Urbina. La Biblioteca Nacional contaba con colecciones heredadas de las bibliotecas coloniales pertenecientes a la Universidad, a los conventos de diversas órdenes religiosas y a los colegios (Fernández de Zamora, 2000, p. 73). La colección había sido organizada en la época que José María Vigil fue su director entre los años 1880 y 1909. Urbina solicitaba en ese entonces un oficial para catalogar y otro para clasificar y en los informes se observaban grandes proyectos a nivel institucional, pero también la insatisfacción por la carencia de respaldo presupuestal para llevar a cabo la obra de rescate y reformas que requería el establecimiento (Quintana Pali, 1988, p. 52). Como es sabido, en 1914 la Biblioteca Nacional de México quedó vinculada a la Universidad Nacional de México y cuando ésta obtuvo su autonomía en 1929, la biblioteca quedó como parte integrante de la UNAM.

En esos años las bibliotecas tenían una importancia enorme en el desarrollo educativo y cultural porque la industria editorial nacional había desarrollado poco la producción masiva de libros. Por lo tanto, se manifiesta desde el gobierno una preocupación por hacer visibles los libros en las bibliotecas mexicanas y para apoyarlas en su trabajo técnico, a través de la Secretaría de Instrucción Pública y Bellas Artes que distribuyó ejemplares de la "Clasificación Decimal de Dewey para bibliotecas: Tablas generales", compendiadas en el año 1914 (Quintana Pali, 1988, p. 36).

En el año de 1915 la dirección de la Biblioteca Nacional es asumida por Luis Manuel Rojas y la subdirección por Agustín Loera y Chávez. La biblioteca se encontraba en un estado lamentable de desorden y había sido sustraído abundante material, debido a lo cual se prestó mucha atención a la reorganización técnica y la clasificación de los libros. Sin embargo, no se tomó la decisión de cambiar el sistema de Namur "hasta tanto no se construyera el sistema de clasificación que a este organismo conviene, para lo cual se llamaría a un grupo de especialistas en cada una de las grandes ramas del saber" (Rojas citado por Quintana Pali, 1988, p. 79). Ésta es una declaración de principios que no vuelve a aparecer en la literatura bibliotecológica.

Es importante destacar que quienes ostentaban la responsabilidad de organizar las colecciones documentales en 1915 tenían claro que era necesario 
construir un sistema de clasificación propio. A continuación del Ateneo de la Juventud surge el Ateneo de México. Entre las otras grandes iniciativas culturales de la época se funda la Sociedad de Conferencias y Conciertos, cuya meta era propagar la cultura entre los estudiantes universitarios de la ciudad de México, y el grupo impulsor era conocido como "los Siete sabios de México", entre los que se contaban personalidades como Alfonso Caso y Vicente Lombardo Toledano. El nuevo grupo intelectual impulsa y permea los ámbitos educativos y culturales incluyendo a la biblioteca y es una fuerte influencia en las personalidades de quienes asumen las responsabilidades administrativas.

En el año de 1917 durante el gobierno carrancista y avalado por la nueva constitución se suprime la Secretaría de Instrucción Pública y Bellas Artes y la Universidad Nacional queda como una de las Direcciones Generales en que fue desmembrada. La idea que se perseguía era la descentralización administrativa de la educación para que los gobiernos municipales fueran los responsables de la misma. Todas las bibliotecas y archivos públicos pasaron a depender de la Universidad Nacional.

El nombramiento de José Vasconcelos como rector de la Universidad Nacional en el año 1920 fue una buena noticia para las bibliotecas, y en palabras de Carlos Monsiváis, "la batalla contra el analfabetismo emprendida por México en 1920 trajo consigo la fe en el libro y la fe en la bibliotecas como eficaces medios de educación popular” (Monsiváis, 1976, p. 346). En 1920 se crea la Dirección de Bibliotecas Populares de la Universidad Nacional, la cual quedó originalmente a cargo de Julio Torri y a partir de 1921 de Carlos Pellicer (Quintana Pali, 1988, p. 127). No se encontraron datos acerca de los sistemas de ordenamiento que utilizaban.

Vasconcelos presentó en octubre de 1920 el "Proyecto de Ley de Creación de la Secretaría de Educación Pública Federal” que fue aprobado en 1921. Junto con todos los establecimientos educativos y culturales, las bibliotecas universitarias, la de la Escuela Nacional Preparatoria y la Nacional pasaban a depender de uno de los tres departamentos creados en la secretaria, que era el Departamento de Bibliotecas, cuyo primer director fue Lombardo Toledano. De las cinco mesas que constituían el departamento, una era la de inventarios y catálogos, que en 1922 dio origen a la sección de la Dirección Central de Bibliografía. Esta dirección a su vez quedó dividida en cuatro secciones, una de las cuales era la de catálogos, a la cual le correspondía reunir los catálogos de todas las bibliotecas de la República, con la que se formarían colecciones de tarjetas bibliográficas clasificadas con el sistema de Melvil Dewey (Valle citado por Quintana Pali, 1988, p. 156). En esa época Lombardo Toledano dictó varias conferencias sobre la clasificación Dewey y 
se publicaron siete años después de la primera edición las "Tablas generales compendiadas del Sistema Decimal de Melvil Dewey".

Se suceden diferentes directores del Departamento de Bibliotecas con pocas referencias a la catalogación y clasificación de las colecciones bibliotecarias, si bien las bibliotecas universitarias seguían dependiendo del mismo. Varias veces en la historia de las bibliotecas de esa época se insiste en el mantenimiento de catálogos para conocer los libros que poseía cada biblioteca dependiente, pero no se explica cómo se desarrollaba esa tarea y si realmente se hacía.

En los siguientes años se destacan algunos bibliotecarios que realizaron una labor fundamental para desarrollar la indización bibliográfica en la universidad y en el país. Entre ellos se destaca Juana Manrique de Lara que había tomado los cursos ofrecidos en la Biblioteca Nacional en 1916 y había quedado a cargo de la sección infantil. Después de iniciada su carrera bibliotecaria recibe una beca para estudiar en la Biblioteca Pública de Nueva York. En 1924 se otorgó el certificado de encargados de biblioteca a Tobías Chávez, quien era el director de la biblioteca de la Escuela de Altos Estudios y a María Teresa Chávez Campomanes quien después de algunos años de trabajar en bibliotecas mexicanas se fue a Estados Unidos a proseguir sus estudios de bibliotecología y posteriormente a trabajar en bibliotecas importantes de ese país. Finalmente, Chávez Campomanes regresó a México para dedicarse a la enseñanza y como complemento de sus clases elaboró varios apuntes de clase sobre catalogación (Sametz, 2010, p. 123-124).

Se identificó un documento que no ha sido citado anteriormente en la literatura bibliotecológica, que muestra la labor titánica de quienes asumieron la responsabilidad de indizar las colecciones de biblioteca, pero también la impotencia ante la pobre respuesta del personal bibliotecario de la universidad. En un informe de la organización que tenía la Jefatura de Bibliotecas y del estado que guardan las diferentes labores, bibliotecas y personal, fechado el 31 de octubre de 1933, firmado por Tobías Chávez explica que había ingresado a la Universidad hacía diez años, como bibliotecario, en la Facultad de Altos Estudios y que más tarde le tocó en suerte ser el fundador de la Sección de la Oficina Central. En este informe explica que:

se está formando un catálogo diccionario general de todos los libros y demás publicaciones pertenecientes a las bibliotecas de la Universidad; en dicho catálogo, que será el catálogo de la Biblioteca de la Universidad se encontrarán registradas las obras existentes catalogadas tanto por su autor, como por su título, y, asimismo, dentro del mismo catálogo se encontrarán agrupados por materias los diversos asuntos de que tratan las obras y aun capítulos de las mismas, así como también artículos de las diversas publicaciones 
periódicas, etc. Además de este catálogo general se está formando en la Oficina Central un catálogo diccionario para cada biblioteca, pues muchas de éstas no cuentan con el personal competente para desarrollar sus labores ni les queda a los empleados tiempo disponible que consagrarle. En la misma Oficina Central se ha clasificado y catalogado totalmente el acervo correspondiente a las bibliotecas de Odontología, de Pedagogía, y de Comercio, estando por lo mismo al corriente los catálogos diccionarios especiales de cada una de estas bibliotecas y el duplicado de todas esas cédulas bibliográficas ya está distribuido por orden alfabético en el catálogo diccionario de la Biblioteca de la Universidad. $^{2}$

En 1934 Juana Manrique de Lara publica en México su Guía de Encabezamientos de Materia para catálogos diccionarios. Es el primer trabajo para apoyar la catalogación por materias realizado en México. A partir de este primer apoyo bibliográfico en materia de temas, para asignar al contenido de los documentos, se publican varias listas que en su mayoría son adaptación y traducción de las listas norteamericanas (Castañón, 1974, p. 182).

En definitiva, para Zamora los procesos técnicos tuvieron dos etapas: la primera iniciada por la Secretaría de Educación Pública a través de su Departamento de Bibliotecas en el año de 1922 con la publicación de tres obras: la ya mencionada sobre la Clasificación de Dewey y otras dos de catalogación y la segunda etapa a partir de la creación de la Biblioteca Franklin creada en 1942 (Zamora, 1985, p. 477).

La Biblioteca Franklin ejerce una influencia definitiva sobre las otras bibliotecas mexicanas al brindar la oportunidad de conocer nuevas herramientas profesionales como el uso de las tarjetas impresas en los catálogos, al igual que en la Biblioteca del Congreso de los Estados Unidos de Norteamérica y el uso del National Union Catalog que vende esta misma biblioteca, además de otros servicios que no interesan a los fines de este trabajo. (Zamora, 1985, p. 478).

A partir de este segundo movimiento bibliotecario, se observa la influencia en la adopción de la clasificación de la Biblioteca del Congreso por la Biblioteca del Museo Nacional de Antropología, a la que le siguen en 1954 las bibliotecas de la UNAM. Además se adoptan en ese mismo año las Reglas de Catalogación de la ALA y posteriormente se introducen los cambios aprobados en París en 1961 (Zamora, 1985, p. 479).

2 Tobías Chávez (1933), Informe de Tobías Chávez, jefe de bibliotecas de la Universidad Nacional de México, relativo a la organización de la jefatura de bibliotecas y del estado que guardan sus diversas labores. Se indican algunas sugerencias para mejorar el servicio. En Fondo Ezequiel A. Chávez, Sec.: Universidad, Subsec.: Rectoría, Serie: Informes 
Seguramente, la circularidad del saber para esos años había influido en las propuestas que comienzan a plantearse en la Bibliotecología. Desde los años veinte se gestaba la Teoría General de la Terminología (influida a su vez por las necesidades de la comunicación científica y técnica), propuesta por el círculo de Viena y Wüster, que proponían entre otras ideas la sistematización de la terminología y la univocidad comunicativa del término científico. Evidentemente en el contexto comunicativo y social de la biblioteca no podía adoptarse esta teoría, que pregonaba la univocidad del término y se plantean repertorios lingüísticos para representar los contenidos documentales, científicos y técnicos, permeados por un espíritu más pragmático.

En este ambiente, en 1961, Gloria Escamilla inicia la recopilación de los encabezamientos de materias, cuando la dirección de la Biblioteca Nacional le encomienda la reorganización del Departamento de Catalogación y Clasificación de dicha institución (Castañón, 1974, p. 184). Como se comenta en la introducción se trata de una recopilación que procede de los principales trabajos anteriores en inglés y castellano y fundamentalmente de la práctica aplicada en la catalogación durante muchos años de los fondos de una biblioteca general. Se publican dos ediciones, una en 1967 y la otra en 1978. México participa en el comité consultor sobre Encabezamientos de Materia celebrado en Washington en 1965. En 1967 la Unión Panamericana publica una lista de encabezamientos de materia compilada bajo la dirección de Carmen Rovira y Jorge Aguayo. Posteriormente en 1985 surge una segunda edición de esta lista bajo los auspicios la OEA y el Instituto Colombiano para el Fomento de la Educación Superior

Las listas de encabezamientos de materia en español no fueron actualizadas con la frecuencia que es obligatoria en una obra de consulta para indización, donde deben estar reflejadas la terminología de los avances científicos y tecnológicos que ocurren cada año.

La característica de las listas de encabezamientos de materia es la alta pre-coordinación de sus encabezamientos porque fueron ideados para catálogos manuales, a mayor cantidad de palabras, mayor especificación de los temas y entradas más directa a la información precisa. Otras de las características son las relaciones asociativas entre encabezamientos y que abarcan todas las ramas del saber humano, porque están elaboradas para sistemas de conocimiento generales como comprenden las bibliotecas públicas o las bibliotecas universitarias.

La Dirección General de Bibliotecas de la UNAM fue creada en 1966; cuenta con una Subdirección Técnica, responsable de las adquisiciones de libros, la catalogación y clasificación del material bibliográfico, el registro y control del acervo de publicaciones periódicas de la UNAM, así como de la 
actualización permanente de los catálogos globales. La asignación de encabezamientos de materia para ofrecer el acceso temático al público usuario la realiza el catalogador mediante la traducción de las propuestas de la Biblioteca del Congreso de los Estados Unidos de Norteamérica. Los catalogadores traducen y usan las listas de encabezamientos en español para interpretar los encabezamientos en inglés, pero la actualización de las listas al español es insuficiente, de acuerdo a la dinámica científica y técnica que está mejor representada en los catálogos en inglés.

La clasificación bibliográfica adoptada es también la que se promueve en la biblioteca mencionada en el párrafo anterior y como consecuencia el ordenamiento en los estantes refleja la visión propia que le otorga la cultura de quienes la realizan. La concepción subyacente en este modo de desarrollar la actividad bibliotecaria es la positivista, de acuerdo con la definición de Moreiro: "formas apriorísticas de representación basadas en categorías universales, preponderancia de las relaciones jerárquicas, documento como soporte del conocimiento, información vista desde la producción o desde la recepción, función pedagógica de la Bibliotecología, difusión de carácter global, sustantivo como forma de representación privilegiada, etc. (Moreiro, 2007, p. 28).

La perspectiva postmoderna, como se verá en el apartado siguiente, va permeando el medio socio-cultural y los modos de organización del conocimiento, en una realidad con sistemas de recuperación sobre un universo digitalizado, que requieren de una respuesta clara y contundente para transferir la información a los usuarios de la misma.

\section{ACCESO TEMÁTICO CONTEMPORÁNEO}

En la actualidad la transferencia electrónica prescinde de recursos de información tangibles como los que se localizan en las bibliotecas en formato físico, sin embargo en la organización de estos recursos se buscan soluciones en los antecedentes bibliotecarios, porque los contenidos son transferidos a través de la lengua y tomando en cuenta las necesidades de los usuarios de información, en un ejercicio de intermediación.

La terminología tiene una relación intrínseca con la Bibliotecología, en la medida que una y otra estudian los términos científicos y tecnológicos para intercambiar información y comunicar los avances del saber.

A su vez, la ingeniería lingüística, la lingüística computacional y la bibliotecología interactúan con la terminología en los ámbitos de la comunicación especializada en el medio digital. De acuerdo a Cabré 
La terminología, concebida como el conjunto de las unidades léxicas usadas con un valor preciso en los ámbitos de especialidad, es el recurso indispensable para representar y comunicar los conocimientos especializados (2010, p. 1).

Si bien la perspectiva positivista ha tenido preponderancia en el quehacer de la indización y acceso a la información desde los años cincuenta del siglo pasado, en el año de 1977 la Bibliotecología comienza a reconocer en el sociocognitivismo un nuevo método de trabajo, desde una concepción contemporánea. Las aproximaciones que se han hecho al tema están recogidos en un artículo de Hjorland (2002, pp. 257-270).

En este mismo campo bibliotecológico Moreiro reconoce que en la postmodernidad "se prefiere destacar la función comunicativa de la lengua, desplazando la intensidad puesta, hasta ahora, en el carácter representativo" (2004, p. 29). Se buscan muchos modos de asociar los términos: jerárquicos, asociativos, conceptuales, explicativos y las listas de encabezamientos se desplazan hacia lenguajes documentales, con mayor flexibilidad (Moreiro, 2004, p. 38) y además tienden a ser recopilados en las lenguas del usuario de la información.

Los tesauros documentales habían aparecido a nivel internacional en la década de los cincuenta y han ido evolucionando de la elaboración manual a la automática, pero siempre con estructuras diseñadas por el bibliotecólogo. Los tesauros documentales a diferencia de las listas de encabezamientos operan en ámbitos especializados del conocimiento e incluyen relaciones jerárquicas, gran cantidad de relaciones asociativas, definiciones para guiar al significado de algún término, explican las equivalencias entre términos, asocian términos entre sí y presentan menor grado de pre-coordinación que las listas de encabezamientos. En la actualidad el discurso ha cambiado hacia la conformación del tesauro conceptual, donde en las jerarquías se incluyen instancias, atributos y meronimias y en el funcionamiento se usan ontologías (Moreiro, 2007, p. 34).

Las normas que rigen la construcción de tesauros se amplían a otros vocabularios controlados como las listas de encabezamientos de materia $u$ ontologías, para organizar información e intercambiar datos estandarizados (norma norteamericana Z39.19:2005, norma británica BS 8723.3:2007 y las ISO 25964-1:2011 Thesauri and interoperability with other vocabularies e ISO/ DIS 25964-2 Interoperability with other vocabularies).

Entre las nuevas tecnologías aparecen las ontologías que ubican mediante métodos computacionales los temas de los contenidos documentales en los documentos digitales, para clasificarlos y permitir la recuperación. Estos mecanismos conocidos como parte integrante de la Web semántica han tenido 
un avance muy lento y su mayor dificultad es la representación lingüística de esos contenidos, porque entre los elementos que sirven de marco, con la finalidad de dar solidez a la estructura de un área de conocimiento en la que se basan las ontologías, se cuentan los instrumentos lingüísticos, utilizados para indizar temas en las bibliotecas (Pastor Sánchez, Martínez Méndes y Rodríguez Muñoz, 2009, p. 73).

Es decir, las ontologías deben recuperar el conocimiento implícito en los documentos, tarea humana por excelencia, para hacerlo explícito en las computadoras (Temmerman, 2003, p. 3). En la actividad humana participan terminólogos, ingenieros lingüistas y lingüistas computacionales. Los bibliotecólogos tienen gran tradición en el trabajo de recuperar la información implícita para hacerla explícita, a través de listas de encabezamientos, listas de palabras claves y tesauros, pero sólo los grandes sistemas de bibliotecas internacionales y servicios de bases de datos han hecho inversiones fuertes en la conformación de instrumentos de indización, con la intervención de los bibliotecólogos.

En paralelo al movimiento bibliotecario y la adaptación a los nuevos modos de transmisión del conocimiento, en la terminología surgen enfoques similares y gana terreno en el trabajo conjunto con los informáticos para desarrollar la calificación de los contenidos digitales y transmitirlos al medio social. La terminología desde fines del siglo pasado está influida por la Teoría Comunicativa de la Terminología y se expresa como un proceso lingüístico documental mediante términos científico-técnicos que son unidades lingüísticas, unidades de conocimiento especializado y unidades de comunicación especializada en una concepción postmoderna del lenguaje (Cabré, 1999, p. 70).

A fines del siglo pasado y comienzos del actual se pregona el nuevo modelo de la Terminología Sociocognitiva, cuya mayor representante es Rita Temmerman. La terminología tradicional consideraba todas las nociones de la misma manera, es decir, según los mismos principios y los mismos métodos. En el cambio, se habla de unidades de comprensión para describir los aspectos flexibles que hacen la variedad de definiciones de un término y que están relacionados con el nivel de especialización del emisor y del receptor del mensaje. Además, se reconoce la polisemia y la sinonimia que ya había adquirido su lugar en la Teoría Comunicativa de la Terminología.

Otro punto importante que ha variado en la terminología es en relación con la inclusión de categorías entre términos, en las cuales se pueden presentar ciertas características en común, pero no necesariamente todas, como se propugnaba desde Aristóteles hasta Wittgenstein. Ellos asumían que en una misma categoría se incluían solamente las cosas que tenían ciertas propiedades en común (Lakoff, 1987, p. 6, citado por Temmerman, 2000, p. 60) 
Sin duda, la terminología juega un papel importante en el concierto de la transferencia de información, pero uno de los aspectos en el que más ha hecho hincapié a diferencia de la Bibliotecología es el trabajo de rescatar los valores culturales que se transmiten a través de la lengua. La literatura sobre terminología marca la necesidad del uso de la propia lengua en la ciencia y la tecnología.

Los escenarios de comunicación especializada forman parte del conjunto de los contextos de comunicación de las sociedades, y por ello una lengua que desee para sí misma un uso normal en intensidad y extensión necesita tener terminología disponible para los usos especializados de sus hablantes en tanto que profesionales y especialistas en temas y sectores concretos (Cabré, 2010, p. 7).

Debido a la circularidad del avance científico y el manejo digital de la información, los instrumentos lingüísticos usados en la bibliotecología para indizar y acceder a la información han reflejado grandes cambios en su pragmatismo, insertados en los nuevos modelos presentes en las ciencias sociales y humanas, influidas por el postmodernismo (Moreiro, 2007, p. 29). Es un fenómeno observable en las normas que rigen la construcción de listas de encabezamientos, tesauros y vocabularios controlados en general, que ya han sido mencionadas líneas arriba. Estas normas internacionales no mencionan la importancia del desarrollo de los instrumentos de indización y acceso en las lenguas de cada país, sin embargo, el discurso de la terminología insiste en su conveniencia y la práctica bibliotecológica lo refuerza, dados los problemas de comunicación observados en los catálogos.

\section{Análisis de la ACtualidad en México}

Las tecnologías ofrecen soluciones físicas, más que conceptuales, para localizar la información que buscan los usuarios. Es aquí donde interesa analizar las decisiones del pasado que afectan el presente.

Como se pudo constatar en el apartado anterior, en la construcción de instrumentos lingüísticos de indización, se propugna por un acceso temático más flexible con relación a la propuesta positivista, que se adopta en las bibliotecas de la UNAM y que manifiesta su influencia aún ahora.

En México se ha trabajado muy poco la conformación de listas de encabezamientos en el español usado en el país y bastante menos en la estructuración de tesauros documentales que hubieran podido ser una opción más generalizada para los catálogos de las colecciones especializadas de la UNAM. 
Los catálogos del sistema bibliotecario de la UNAM muestran un español traducido, que no refleja tampoco los usos lingüísticos del español usado en México (Naumis, 2009, p. 67). La traducción es puntual, la realiza el catalogador cuando estructura el registro bibliográfico de una obra y no existe un trabajo consensuado de aceptación del lenguaje que transmitirán los catálogos al usuario. ${ }^{3}$

La parte más difícil es hallar el equilibrio entre traducción y adaptación. Según Delisle y Bastin en la adaptación global se hacen numerosos cambios importantes, de manera que ya no se puede hablar de una traducción. La adaptación puntual, sin embargo, se limita a algunas partes del discurso y se utiliza, por ejemplo, cuando la realidad a la que se remite la obra original difiere mucho de la realidad a la que se remite la traducción, es decir, cuando en una cultura no existe un concepto que sí existe en otra (2006: p. 175). Los términos que se han traducido en México no están siempre adaptados a los términos significativos en el país y reflejan en buena medida la idiosincrasia y relaciones sociales existentes en Estados Unidos de Norteamérica (Naumis, 2009, p. 67-84).

Los antecedentes indican el riesgo de que el acceso temático hacia sistemas de información en México, usando la Web semántica, se realice en el español no usado en México. La bibliotecología por sí sola podría hacer poco en este sentido, sin embargo la conjunción con otras áreas del conocimiento, que comparten necesidades similares en el ámbito sociocultural y disciplinario donde se desarrollan, es una posibilidad para trabajar en lenguajes de indización y recuperación en nuestra propia lengua.

\section{CONCLUSIONES Y PERSPECTIVAS}

Se puede afirmar, según los datos recogidos, que cuando se crea la Universidad Nacional y comienzan a desarrollarse sus bibliotecas no se continuó impulsando un esfuerzo que había comenzado a fines del siglo XIX y principios del XX

3 Por ejemplo, el catálogo de autoridades de Librunam enlista entre los encabezamientos: Pluralismo cultural, con una clara influencia del inglés Cultural pluralism. En México, los términos utilizados por la comunidad científica son Diversidad Cultural, que no aparece como encabezamiento aceptado, y Multiculturalismo, que se incluye en el registro de autoridad como término asociado. En la propia UNAM existen el Programa Universitario México, Nación Multicultural y el Seminario Sociedad del Conocimiento y Diversidad Cultural, este último forma parte de las redes de investigación del Conacyt. El Consejo Nacional de Población de la Secretaría de Gobernación promueve una Campaña nacional por la diversidad cultural de México. Obtenido el 16 de marzo de 2012.

http://132.248.67.3:8991/F/9JEUF3E1DL99KGISSNBEDMRI19GEIC3AP3D3R8L7SE9YM7DDJL-00223? func $=$ find-b-0\&local_base $=$ MX011\# 
por integrarse en las decisiones bibliográficas promovidas desde Europa, como lo demuestra el poco interés de Justo Sierra para continuar con la participación en las reuniones bibliográficas.

Los testimonios explican la adhesión a la Clasificación Decimal Dewey surgida en Estados Unidos, que por un tiempo se utilizó con las adecuaciones europeas. Sin embargo, poco a poco se fue privilegiando el uso de la clasificación estadounidense original y abandonando lo que posteriormente sería la Clasificación Decimal Universal.

La primera mitad del siglo XX fueron años de intenso trabajo para quienes tenían la responsabilidad de echar a andar las bibliotecas y no se les puede criticar los modelos que adoptaron. A lo largo del siglo XX se mostró la utilidad de la praxis americana para organizar bibliotecas y la opción de traducir sus encabezamientos y utilizar sus sistemas clasificatorios.

La historia del acceso temático en las bibliotecas de la UNAM muestra un panorama que quizás no difiera de otros países de habla hispana, pero testimonia el uso de herramientas externas traducidas de otros idiomas, que responden a modelos obsoletos de acuerdo a las tendencias modernas.

En el marco del procesamiento catalográfico de la UNAM durante la segunda mitad del siglo XX predominó la adhesión a la catalogación por materias mediante la traducción del inglés al español, de los encabezamientos de materia utilizados en la Library of Congress de Estados Unidos de Norteamérica. Se adoptó la Clasificación de la misma biblioteca americana, ubicando los contenidos de los libros directamente en las jerarquías de su sistema de clasificación. Los sistemas bibliográficos clasificatorios son de tipo pragmático y, sin duda, están concebidos en un ámbito cultural que permea las decisiones clasificatorias e influye en el medio que las recibe, al ordenar el material bibliográfico con los parámetros de otra sociedad.

Como objetos precisos y unificados como son los términos y de un vehículo como es el documental, no solucionan el problema de la representación y comunicación temática en un ámbito especializado, porque resolverlo supone la obtención de términos representativos del universo a describir. La traducción de términos como se ha realizado tradicionalmente no es la solución adecuada, más bien es necesaria la conversión de las situaciones comunicativas de los documentos en el medio social donde se difundirán.

Desde la Teoría Sociocognitiva de la Terminología se recomienda que se construyan sistemas de acceso a la información basados en la cultura propia y la traducción debe ser utilizada como guía, pero siempre adaptada a los valores sociales del grupo al que se dirige. Ello sólo se puede hacer mediante estudios lingüísticos mucho más profundos que sólo categorizar el material con decisiones rápidas y personales tomadas en el momento de la catalogación. 
Se debe desarrollar a los fines de organización de contenidos documentales la investigación lingüística aplicada a la comunicación científica y tecnológica en nuestro propio idioma, tanto desde su utilización para interpretar otros idiomas, como para proponer modelos culturales, científicos y tecnológicos propios que se transmiten a través de la lengua.

\section{OBRAS CONSUlTADAS}

ANSI/NISO Z39.19.2005. Guidelines for the construction, format and management of monolingual controlled vocabularies. ( $4^{\mathrm{a}} \mathrm{ed}$.) Bethesda, Md: National Information Standards Organizationa, 2005.

Astrain Gallart, M., Olagûe de Ros, G. y Menéndez Navarro, A. (2001) "Ciencia y documentación científica en la periferia. La Royal Society y la creación de la oficina bibliográfica mexicana (1895-1929)" En Asclepio, 53(1), 295- 312

Cabré Castellví, M. T. (1999) La terminología: representación y comunicación: elementos para una teoría de base comunicativa y otros artículos. Barcelona, IULA. (2010) “Terminología y normalización lingüística”. p. 1-19 Obtenida el 19 de enero de 2012 de http://www.ei.ehu.es/p05612532/eu/contenidos/informacion/euskara_inst_jardunaldiak2002/eu_jard2002/adjuntos/01.pdf

Caso, Antonio (1943) Apuntamientos de cultura patria. México.

Castañón Moreno, B. (1974) Los encabezamientos de materia. México: La autora. 238 h. Tesis (Licenciatura en Bibliotecología) - UNAM

Delisle, J. y Bastin, G. L (2006) Iniciación a la traducción: enfoque interpretativo: teoría y práctica. Caracas: Univ. Central de Venezuela, 291 p. (Colección Estudios)

Escamilla González, G. (1970) “Los encabezamientos de materia”. En Boletín de la Biblioteca Nacional de México, 3 (ene-jun), 181-194

Fernández de Zamora, R. M. (2000) "La Biblioteca Nacional de México hacia el nuevo siglo”. En Métodos de información, 7 (40), $72-77$

Hjorland, B. (2002) "Epistemology and the Socio-Cognitive Perspective in Information Science". En Journal of the American Society for Informationa Science and Technology, 53(4), 257-270)

Iguíniz, J. B. (1950) "La Biblioteca Nacional de México". En Boletín de la Biblioteca Nacional de México, 2ª Época, tomo 1(1), 5-28

Mantecón Navasal, J. I. (1975) "Notas para una bibliografía de reglas y listas de encabezamientos de materia en español". Tirada aparte del trabajo publicado en el libro Homenaje a Don Agustín Millares Carlo. Caja Insular de Ahorros de Gran Canaria.

Monsiváis, C. (1976) "Notas sobre la cultura mexicana en el siglo XX". En Historia General de México. México: El Colegio de México. Tomo 4. 
Morales Campos, E. (2010) "Sociedades letradas y cultura impresa en México a principios del siglo XX". En Jornadas Mexicanas de Biblioteconomía (41, 3-5 de mayo de 2010) Obtenida el 12 de octubre del 2010 http://ambac.org.mx/amate/es/jornadas/ponencias/ descargar/118/161

Moreiro González, J. A. "Evolución paralela de los Lenguajes Documentales y la Terminología”. En La interdisciplinariedad y la transdisciplinariedad en la organización del conocimiento científico = Interdisciplinarity and transdisciplinarity in the organization of scientific knowledge Actas del VIII Congreso ISKO-España. León, 18, 19 y 20 de abril de 2007. pp. 27-42.

Naumis Peña, Catalina (2009) "La traducción para indizar los contenidos de los recursos bibliográficos digitales”. p. 49-69. En Memorias del Coloquio de Investigación Bibliotecológica y sobre la información (26: 1-3 octubre 2008: México DF) / comp. F. Martínez Arellano y J. J. Calva González. -- México: CUIB.

Pastor Sánchez, J. A., Martínez Méndes F. J. y Rodríguez Muñoz J. V. (2009) "Advantages of thesaurus representation using the Simple Knowledge Organization System (SKOS) compared with proposed alternatives". En Information Research, 4 (14) Obtenido el 10 de agosto de 2011 en http://InformationR.net/ir/14-4/paper422.ht$\mathrm{ml}]$

Quintana Pali, G. (1986) "La investigación histórica sobre las bibliotecas públicas del Distrito Federal durante los años de 1910 a 1940". En Jornadas Mexicanas de Biblioteconomía (16: 6 al 10 de mayo de 1985, Pachuca, Hidalgo). p. 293-297-

Quintana Pali, G, Gil Villegas, C. y Tolosa Sánchez, G. (1988) Las bibliotecas públicas en México: 1910-1940. México: SEP, Dirección General de Bibliotecas. 406 p. (Historia de las bibliotecas en México;3)

Organización de Estados Iberoamericanos para la Educación, la Ciencia y la Cultura. Sistemas Nacionales de Cultura. Informe de México. Establecimientos e instituciones culturales ('Terminado en el 2002) Obtenido el 12 de octubre de 2010 en http://www.oei. es/cultura2/mexico/c8b.htm

Sametz, L. (2010) Vasconcelos el hombre del libro: creador del primer sistema de bibliotecas. México: Conaculta.

Temmerman, R. (2003) "Termonotography: Ontology Building and the Sociocognitive Approach to Terminology Description”. In Proceedings of the XVIII International Congress of Linguistics (24-29 July), Prague: University Karlova (CD- ROM).

(2000) "Une théorie réaliste de la terminologie: le sociocognitivisme”. En Terminologies Nouvelles, 21, 58-64.

Zamora R., P. (1985) "Mesa redonda: comentarios de los expresidentes de la AMBAC". p. En Jornadas Mexicanas de Biblioteconomía, (15, 30 de abril al 4 de mayo 1984, Tlaxcala) p. 477-481. 
(1955) "Nueva actitud hacia las reglas de catalogación" En Boletín de la Biblioteca Nacional, 2a . Época, tomo 6 (3), p. 3242

$\infty$ 